\title{
Light vector meson masses in strange hadronic matter - a QCD sum rule approach
}

\author{
Amruta Mishra* \\ Department of Physics, Indian Institute of Technology, \\ Delhi, Hauz Khas, New Delhi - 110 016, India
}

\begin{abstract}
We study the properties of the light vector mesons $(\rho, \omega$ and $\phi)$ in strange hadronic matter using the QCD sum rule approach. The in-medium masses of the vector mesons are calculated from the modifications of the light quark condensates and the gluon condensates in the hadronic medium. The light quark condensates in the hadronic matter are obtained from the values of the non strange and strange scalar fields of the explicit chiral symmetry breaking term in a chiral SU(3) model. The in-medium gluon condensate is calculated through the medium modification of a scalar dilaton field, which is introduced into the chiral SU(3) model to simulate the scale symmetry breaking of QCD. The mass of the $\omega$ meson is observed to have initially a drop with increase in density and then a rise due to the scattering with the baryons. The mass of the $\rho$ meson is seen to drop with density due to decrease of the light quark condensates in the medium. The effects of isospin asymmetry and strangeness of the medium on the masses of the vector mesons are also studied in the present work. The $\phi$ meson is observed to have marginal drop in its mass in the nuclear medium. However, the strangeness of the medium is seen to lead to an appreciable increase in its mass arising due to scattering with the hyperons.
\end{abstract}

*Electronic address: amruta@physics.iitd.ac.in 


\section{INTRODUCTION}

The study of the properties of hadrons in hot and dense matter is an important topic of research in strong interaction physics. The changes in the hadron properties in the medium affect the experimental observables from the hot and/or dense matter produced in the heavy ion collision experiments. The medium modifications of the properties of the light vector

mesons [1] can affect the low mass dilepton spectra, the properties of the kaons and antikaons can show in their production as well as collective flow. The modifications of the properties of the charm mesons, $D$ and $\bar{D}$ as well as the charmonium states can modify the yield of the open charm meson as well as charmonium states in the high energy nuclear collision experiments.

In the present work, we study the medium modification of the masses of the light vector mesons $(\rho, \omega$ and $\phi)$ in the strange hadronic matter due to the interaction with the light quark condensates and the gluon condensates using the QCD sum rule approach [2-7]. The light quark condensates are calculated from the expectation values of the non-strange and strange scalar fields of the explicit chiral symmetry breaking term in a chiral SU(3) model [8, 9]. The gluon condensate in the hadronic medium is obtained from the medium modification of a scalar dilaton field introduced within the chiral SU(3) model through a scale symmetry breaking term in the Lagrangian density leading to the QCD trace anomaly. The chiral SU(3) model has been used to describe the hadronic properties in the vacuum as well as in nuclear matter [8], finite nuclei [9] and the bulk properties of (proto) neutron stars [10]. The vector mesons have also been studied within the model [11], arising due to their interaction with the nucleons in the medium. The model has been used to study the medium modifications of kaons and antikaons in isospin asymmetric nuclear matter in [12] and in hyperonic matter in [13]. The chiral effective model has also been generalized to SU(4) to derive the interactions of the charm mesons with the light hadrons to study the $D$ mesons in asymmetric nuclear matter at zero temperature [14] and in the symmetric and asymmetric nuclear (hyperonic) matter at finite temperatures in Ref.[15] and Ref. [16, 17]. In the present investigation, we study the light vector mesons using QCD sum rule approach due to their interaction with the quark and gluon condensates in the strange hadronic medium. These in-medium condensates are calculated in a chiral SU(3) model, from the explicit 
chiral symmetry breaking term and the scale breaking term of the Lagrangian density of the effective hadronic model.

The outline of the paper is as follows : In section II, we give a brief introduction of the chiral $S U(3)$ model used to calculate the quark and gluon condensates in the hadronic medium. In the present work, the in-medium condensates as calculated in the chiral SU(3) model, are taken as inputs for studying the in-medium masses of the light vector mesons using the QCD sum rule approach. The medium modifications of the quark and gluon condensates arise from the medium modification of the scalar fields of the explicit symmetry breaking term and of the scalar dilaton field introduced in the hadronic model to incorporate broken scale invariance of QCD. In section III, we present the results for the medium modifications of the light vector mesons using a QCD sum rule approach. In section IV, we summarize the findings of the present investigation and compare with the existing results in the literature for the in-medium properties of the light vector mesons.

\section{THE HADRONIC CHIRAL $S U(3) \times S U(3)$ MODEL}

We use an effective chiral $S U(3)$ model for the present investigation [9]. The model is based on the nonlinear realization of chiral symmetry [18 20] and broken scale invariance [8, 9, 11]. This model has been used successfully to describe nuclear matter, finite nuclei, hypernuclei and neutron stars. The effective hadronic chiral Lagrangian density contains the following terms

$$
\mathcal{L}=\mathcal{L}_{\text {kin }}+\sum_{W=X, Y, V, A, u} \mathcal{L}_{B W}+\mathcal{L}_{\text {vec }}+\mathcal{L}_{0}+\mathcal{L}_{S B}
$$

In Eq. (11) $\mathcal{L}_{k i n}$ is kinetic energy term, $\mathcal{L}_{B W}$ is the baryon-meson interaction term in which the baryon-spin-0 meson interaction term generates the vacuum baryon masses. $\mathcal{L}_{\text {vec }}$ describes the dynamical mass generation of the vector mesons via couplings to the scalar mesons and contain additionally quartic self-interactions of the vector fields. $\mathcal{L}_{0}$ contains the meson-meson interaction terms inducing the spontaneous breaking of chiral symmetry as well as a scale invariance breaking logarithmic potential. $\mathcal{L}_{S B}$ describes the explicit chiral symmetry breaking. 
To study the in-medium hadron properties using the chiral SU(3) model, we use the mean field approximation, where all the meson fields are treated as classical fields. In this approximation, only the scalar and the vector fields contribute to the baryon-meson interaction, $\mathcal{L}_{B W}$ since for all the other mesons, the expectation values are zero. The baryonscalar meson coupling constants are fitted from the vacuum masses of the baryons. The parameters in the model [9, 12] are chosen so as to decouple the strange vector field $\phi_{\mu} \sim \bar{s} \gamma_{\mu} s$ from the nucleon.

The concept of broken scale invariance leading to the trace anomaly in $\mathrm{QCD}, \theta_{\mu}^{\mu}=$ $\frac{\beta_{Q C D}}{2 g} G^{a}{ }_{\mu \nu} G^{\mu \nu a}$, where $G_{\mu \nu}^{a}$ is the gluon field strength tensor of QCD, is simulated in the effective Lagrangian at tree level through the introduction of the scale breaking terms [21, 22]

$$
\mathcal{L}_{\text {scalebreaking }}=-\frac{1}{4} \chi^{4} \ln \left(\frac{\chi^{4}}{\chi_{0}^{4}}\right)+\frac{d}{3} \chi^{4} \ln \left(\left(\frac{\sigma^{2} \zeta}{\sigma_{0}^{2} \zeta_{0}}\right)\left(\frac{\chi}{\chi_{0}}\right)^{3}\right) .
$$

The Lagrangian density corresponding to the dilaton field, $\chi$ leads to the trace of the energy momentum tensor as [23, 24]

$$
\theta_{\mu}^{\mu}=\chi \frac{\partial \mathcal{L}}{\partial \chi}-4 \mathcal{L}=-(1-d) \chi^{4}
$$

The comparison of the trace of the energy momentum tensor arising from the trace anomaly of QCD with that of the present chiral model given by equation (3), gives the relation of the dilaton field to the scalar gluon condensate. We have, in the limit of finite quark masses [25],

$$
T_{\mu}^{\mu}=\sum_{i=u, d, s} m_{i} \bar{q}_{i} q_{i}+\left\langle\frac{\beta_{Q C D}}{2 g} G_{\mu \nu}^{a} G^{\mu \nu a}\right\rangle \equiv-(1-d) \chi^{4},
$$

where the first term of the energy-momentum tensor, within the chiral SU(3) model is the negative of the explicit chiral symmetry breaking term, $\mathcal{L}_{S B}$. In the mean field approximation, this chiral symmetry breaking term is given as

$$
\mathcal{L}_{S B}=\operatorname{Tr}\left[\operatorname{diag}\left(-\frac{1}{2} m_{\pi}^{2} f_{\pi}(\sigma+\delta),-\frac{1}{2} m_{\pi}^{2} f_{\pi}(\sigma-\delta),\left(\sqrt{2} m_{k}^{2} f_{k}-\frac{1}{\sqrt{2}} m_{\pi}^{2} f_{\pi}\right) \zeta\right)\right] .
$$

In the above, we have explicitly written down the matrix whose trace gives the Lagrangian density corresponding to the explicit chiral symmetry breaking in the chiral $\mathrm{SU}(3)$ model. Comparing the above term with the explicit chiral symmetry breaking term of the 
Lagrangian density in QCD given as

$$
\mathcal{L}_{S B}^{Q C D}=-\operatorname{Tr}\left[\operatorname{diag}\left(m_{u} \bar{u} u, m_{d} \bar{d} d, m_{s} \bar{s} s\right)\right]
$$

we obtain the nonstrange quark condensates $(\langle\bar{u} u\rangle$ and $\langle\bar{d} d\rangle)$ and the strange quark condensate $(\langle\bar{s} s\rangle)$ to be related to the the scalar fields, $\sigma, \delta$ and $\zeta$ as

$$
\begin{aligned}
& m_{u}\langle\bar{u} u\rangle=\frac{1}{2} m_{\pi}^{2} f_{\pi}(\sigma+\delta) \\
& m_{d}\langle\bar{d} d\rangle=\frac{1}{2} m_{\pi}^{2} f_{\pi}(\sigma-\delta)
\end{aligned}
$$

and,

$$
m_{s}\langle\bar{s} s\rangle=\left(\sqrt{2} m_{k}^{2} f_{k}-\frac{1}{\sqrt{2}} m_{\pi}^{2} f_{\pi}\right) \zeta .
$$

The coupled equations of motion for the non-strange scalar isoscalar field $\sigma$, scalar isovector field, $\delta$, the strange scalar field $\zeta$, and the dilaton field $\chi$, derived from the Lagrangian density, are solved to obtain the values of these fields in the strange hadronic medium.

The QCD $\beta$ function occurring in the right hand side of equation (4), at one loop level, for $N_{c}$ colors and $N_{f}$ flavors, is given as

$$
\beta_{\mathrm{QCD}}(g)=-\frac{11 N_{c} g^{3}}{48 \pi^{2}}\left(1-\frac{2 N_{f}}{11 N_{c}}\right)+O\left(g^{5}\right)
$$

We then obtain the trace of the energy-momentum tensor in QCD, using the one loop beta function given by equation (10), for $N_{c}=3$ and $N_{f}=3$, as given by,

$$
\theta_{\mu}^{\mu}=-\frac{9}{8} \frac{\alpha_{s}}{\pi} G_{\mu \nu}^{a} G^{\mu \nu a}+\left(m_{\pi}^{2} f_{\pi} \sigma+\left(\sqrt{2} m_{k}^{2} f_{k}-\frac{1}{\sqrt{2}} m_{\pi}^{2} f_{\pi}\right) \zeta\right)
$$

where $\alpha_{s}=\frac{g^{2}}{4 \pi}$. Using equations (41) and (11), we can write

$$
\left\langle\frac{\alpha_{s}}{\pi} G^{a}{ }_{\mu \nu} G^{a \mu \nu}\right\rangle=\frac{8}{9}\left[(1-d) \chi^{4}+\left(m_{\pi}^{2} f_{\pi} \sigma+\left(\sqrt{2} m_{k}^{2} f_{k}-\frac{1}{\sqrt{2}} m_{\pi}^{2} f_{\pi}\right) \zeta\right)\right] .
$$

Hence the scalar gluon condensate of QCD $\left(\left\langle G^{a}{ }_{\mu \nu} G^{\mu \nu a}\right\rangle\right)$ is simulated by a scalar dilaton field in the present hadronic model. For the case of massless quarks, the scalar gluon condensate is proportional to the fourth power of the dilaton field, whereas for the case of finite masses of quarks, there are modifications arising from the scalar fields, $\sigma$ and $\zeta$. 
We calculate the light quark condensates, $\langle\bar{u} u\rangle,\langle\bar{d} d\rangle$ and $\langle\bar{s} s\rangle$ and the scalar gluon condensate, $\left\langle\frac{\alpha_{s}}{\pi} G^{a}{ }_{\mu \nu} G^{a \mu \nu}\right\rangle$ in the hadronic medium using the equations (7), (8) and (9) and (12) respectively, from the medium modifications of the scalar fields, $\sigma, \delta, \zeta$ and $\chi$. These values of the quark and gluon condensates are then taken as inputs for the studying the masses of the light vector mesons $(\omega, \rho, \phi)$ in the strange hadronic matter using the QCD sum rule approach. In the next section we shall describe the QCD sum rule approach to study these in-medium vector meson masses in the isospin asymmetric strange hadronic medium.

\section{QCD SUM RULE APPROACH}

In the present section, we investigate the properties of the light vector mesons $(\omega, \rho, \phi)$ in the nuclear medium using the method of QCD sum rules. The in-medium masses of the vector mesons are computed from the medium modifications of the light quark condensates and the scalar gluon condensate calculated in the chiral effective model as described in the previous section. The current current correlation function for the vector meson, $\mathrm{V}(=\omega, \rho, \phi)$ is written as

$$
\Pi_{\mu \nu}=i \int d^{4} x d^{4} y\left\langle 0\left|T j_{\mu}^{V}(x) j_{\nu}^{V}(0)\right| 0\right\rangle,
$$

where $T$ is the time ordered product and $J_{\mu}^{V}$ is the current for the vector meson, $V=\rho, \omega, \phi$,

given as $j_{\mu}^{\rho}=\frac{1}{2}\left(\bar{u} \gamma_{\mu} u-\bar{d} \gamma_{\mu} d\right), j_{\mu}^{\omega}=\frac{1}{6}\left(\bar{u} \gamma_{\mu} u+\bar{d} \gamma_{\mu} d\right)$ and $j_{\mu}^{\phi}=-\frac{1}{3}\left(\bar{s} \gamma_{\mu} s\right)$. Current conservation gives the transverse tensor structure for the correlation function as

$$
\Pi_{\mu \nu}^{V}(q)=\left(g_{\mu \nu}-\frac{q_{\mu} q_{\nu}}{q^{2}}\right) \Pi^{V}\left(q^{2}\right)
$$

where,

$$
\Pi^{V}\left(q^{2}\right)=\frac{1}{3} g^{\mu \nu} \Pi_{\mu \nu}^{V}(q) .
$$

The correlation function $\Pi^{V}\left(q^{2}\right)$ in the large space-like region $Q^{2}=-q^{2}>>1 \mathrm{GeV}^{2}$ for the light vector mesons $(\omega, \rho$ and $\phi)$ can be written in terms of the operator product expansion (OPE) as [5, 6]

$$
12 \pi^{2} \tilde{\Pi}^{V}\left(q^{2}=-Q^{2}\right)=d_{V}\left[-c_{0}^{V} \ln \left(\frac{Q^{2}}{\mu^{2}}\right)+\frac{c_{1}^{V}}{Q^{2}}+\frac{c_{2}^{V}}{Q^{4}}+\frac{c_{3}^{V}}{Q^{6}}+\cdots\right]
$$


where, $\tilde{\Pi}^{V}\left(q^{2}=-Q^{2}\right)=\frac{\Pi^{V}\left(q^{2}=-Q^{2}\right)}{Q^{2}}$ and $\mu$ is a scale which we shall take as $1 \mathrm{GeV}$ in the present investigation. The coefficients $c_{i}^{V}$ 's in equation (16) contain the informations of the nonperturbative effects of QCD in terms of the quark and gluon condensates. In equation (16) $, d_{V}=3 / 2,1 / 6$ and $1 / 3$, for $\rho, \omega$ and $\phi$ vector mesons respectively.

For the vector mesons, $\rho$ and $\omega$, containing the $\mathrm{u}$ and $\mathrm{d}$ quarks (antiquarks), these coefficients are given as [5]

$$
\begin{aligned}
c_{0}^{(\rho, \omega)} & =1+\frac{\alpha_{s}\left(Q^{2}\right)}{\pi}, \quad c_{1}^{(\rho, \omega)}=-3\left(m_{u}^{2}+m_{d}^{2}\right) \\
c_{2}^{(\rho, \omega)} & =\frac{\pi^{2}}{3}\left\langle\frac{\alpha_{s}}{\pi} G^{\mu \nu} G_{\mu \nu}\right\rangle+4 \pi^{2}\left\langle m_{u} \bar{u} u+m_{d} \bar{d} d\right\rangle \\
c_{3}{ }^{(\rho, \omega)} & =-4 \pi^{3}\left[\left\langle\alpha_{s}\left(\bar{u} \gamma_{\mu} \gamma_{5} \lambda^{a} u \mp \bar{d} \gamma_{\mu} \gamma_{5} \lambda^{a} d\right)^{2}\right\rangle\right. \\
& \left.+\frac{2}{9}\left\langle\alpha_{s}\left(\bar{u} \gamma_{\mu} \lambda^{a} u+\bar{d} \gamma_{\mu} \lambda^{a} d\right)\left(\sum_{q=u, d, s} \bar{q} \gamma^{\mu} \lambda^{a} q\right)\right\rangle\right]
\end{aligned}
$$

In the above, $\alpha_{S}=4 \pi /\left(b \ln \left(Q^{2} / \Lambda_{Q C D}{ }^{2}\right)\right)$ is the running coupling constant, with $\Lambda_{Q C D}=140 \mathrm{MeV}$ and $b=11-(2 / 3) N_{f}=9$. In equation (19), the ' $\mp$ ' sign in the first term corresponds to $\rho(\omega)$ meson.

For $\phi$ meson, these coefficients are given as [5, 26]

$$
\begin{gathered}
c_{0}^{\phi}=1+\frac{\alpha_{s}\left(Q^{2}\right)}{\pi}, \quad c_{1}^{\phi}=-6 m_{s}{ }^{2} \\
c_{2}^{\phi}=\frac{\pi^{2}}{3}\left\langle\frac{\alpha_{s}}{\pi} G^{\mu \nu} G_{\mu \nu}\right\rangle+8 \pi^{2}\left\langle m_{s} \bar{s} s\right\rangle \\
c_{3}{ }^{\phi}=-8 \pi^{3}\left[2\left\langle\alpha_{s}\left(\bar{s} \gamma_{\mu} \gamma_{5} \lambda^{a} s\right)^{2}\right\rangle+\frac{4}{9}\left\langle\alpha_{s}\left(\bar{s} \gamma_{\mu} \lambda^{a} s\right)\left(\sum_{q=u, d, s} \bar{q} \gamma^{\mu} \lambda^{a} q\right)\right\rangle\right]
\end{gathered}
$$

After Borel transformation, the correlator for the vector meson given by equation (16) can be written as

$$
12 \pi^{2} \tilde{\Pi}^{V}\left(M^{2}\right)=d_{V}\left[c_{0}^{V} M^{2}+c_{1}^{V}+\frac{c_{2}^{V}}{M^{2}}+\frac{c_{3}^{V}}{2 M^{4}}\right]
$$

On the phenomenological side, the correlator function, $\tilde{\Pi}^{V}\left(Q^{2}\right)$ can be written as

$$
12 \pi^{2} \tilde{\Pi}_{\text {phen }}^{V}\left(Q^{2}\right)=\int_{0}^{\infty} d s \frac{R_{\text {phen }}^{V}(s)}{s+Q^{2}}
$$


where $R_{\text {phen }}^{V}(s)$ is the spectral density proportional to the imaginary part of the correlator

$$
R_{\text {phen }}^{V}(s)=12 \pi \operatorname{Im} \Pi_{\text {phen }}^{V}(s) .
$$

On Borel transformation, equation (24) reduces to

$$
12 \pi^{2} \tilde{\Pi}^{V}\left(M^{2}\right)=\int_{0}^{\infty} d s e^{-s / M^{2}} R_{p h e n}^{V}(s)
$$

Equating the correlation functions from the phenomenological side given by equation (26) to that from the operator product expansion given by equation (23), we obtain,

$$
\int_{0}^{\infty} d s e^{-s / M^{2}} R_{\text {phen }}^{V}(s)=d_{V}\left[c_{0}^{V} M^{2}+c_{1}^{V}+\frac{c_{2}^{V}}{M^{2}}+\frac{c_{3}^{V}}{2 M^{4}}\right] .
$$

The finite energy sum rules (FESR) for the vector mesons are derived from equation (27) by assuming that the spectral density separates to a resonance part $R_{p h e n}^{V(r e s)}(s)$ with $s \leq s_{0}^{V}$ and a perturbative continuum as

$$
R_{\text {phen }}^{V}(s)=R_{\text {phen }}^{V(r e s)}(s) \theta\left(s_{0}^{V}-s\right)+d_{V} c_{0}^{V} \theta\left(s-s_{0}^{V}\right)
$$

For $M>\sqrt{s_{0}^{V}}$, the exponential function in the integral of the left hand side of the equation (27) can be expanded in powers of $s / M^{2}$ for $s<s_{0}^{V}$. We then obtain the left hand side of equation (27) as

$$
\begin{aligned}
& \int_{0}^{\infty} e^{-s / M^{2}} R_{\text {phen }}^{V}(s)=\int_{0}^{s_{0}^{V}} d s R_{\text {phen }}^{V^{(r e s)}}(s)-\frac{1}{M^{2}} \int_{0}^{s_{0}^{V}} d s s R_{\text {phen }}^{V^{(r e s)}}(s)+\frac{1}{2 M^{4}} \int_{0}^{s_{0}^{V}} d s s^{2} R_{\text {phen }}^{V^{(r e s)}}(s) \\
+ & d_{V} c_{0} M^{2}\left(1-\frac{s_{0}^{V}}{M^{2}}+\frac{\left(s_{0}^{V}\right)^{2}}{2 M^{4}}+\frac{\left(s_{0}^{V}\right)^{3}}{6 M^{6}}-\cdots\right)
\end{aligned}
$$

Equating the powers in $1 / M^{2}$ in the Borel transformations of the spectral functions, given by equations (28) and (29), we obtain the Finite energy sum rules (FESR) as

$$
\begin{gathered}
\int_{0}^{s_{0}^{V}} d s R_{p h e n}^{V^{(r e s)}}=d_{V}\left(c_{0}^{V} s_{0}^{V}+c_{1}^{V}\right) \\
\int_{0}^{s_{0}^{V}} d s s R_{p h e n}^{V^{(r e s)}}=d_{V}\left(\frac{\left(s_{0}^{V}\right)^{2} c_{0}^{V}}{2}-c_{2}^{V}\right) \\
\int_{0}^{s_{0}^{V}} d s s^{2} R_{\text {phen }}^{V(r e s)}=d_{V}\left(\frac{\left(s_{0}^{V}\right)^{3}}{3} c_{0}^{V}+c_{3}^{V}\right)
\end{gathered}
$$


To evaluate $c_{3}^{V}$ for the vector mesons $\rho, \omega$ and $\phi$, given by equations (19) and (22), we use factorization method [27],

$$
\left\langle\left(\bar{q}_{i} \gamma_{\mu} \gamma_{5} \lambda^{a} q_{j}\right)^{2}\right\rangle=-\left\langle\left(\bar{q}_{i} \gamma_{\mu} \lambda^{a} q_{j}\right)^{2}\right\rangle=\delta_{i j} \frac{16}{9} \kappa_{i}\left\langle\bar{q}_{i} q_{i}\right\rangle^{2}
$$

for $q_{i}=u, d, s$ for $i=1,2,3$. In the above, $\kappa_{i}$ is introduced to parametrise the deviation from exact factorization $\left(\kappa_{i}=1\right)$. Using equation (33), the four quark condensate for the $\omega(\rho)$ meson given by equation (19) becomes

$$
c_{3}{ }^{(\rho, \omega)}=-\alpha_{s} \pi^{3} \times \frac{448}{81} \kappa_{q}\left(\langle\bar{u} u\rangle^{2}+\langle\bar{d} d\rangle^{2}\right),
$$

where we have used, $\kappa_{u} \simeq \kappa_{d}=\kappa_{q}$.

For the $\phi$ meson, using equations (22) and (33), we obtain the four quark condensate, $c_{3}{ }^{\phi}$ as given by [26]

$$
c_{3}{ }^{\phi}=-8 \pi^{3} \times \frac{224}{81} \alpha_{s} \kappa_{s}\langle\bar{s} s\rangle^{2} .
$$

We assume a simple ansatz for the spectral function, $R_{\text {phen }}^{V}(s)$ as [5, 6]

$$
R_{\text {phen }}^{V}(s)=F_{V} \delta\left(s-m_{V}^{2}\right)+d_{V} c_{0}^{V} \theta\left(s-s_{0}^{V}\right)
$$

Using the form of the spectral function given by equation (36), the finite energy sum rules for vacuum given by equations (30) to (32), can be written as

$$
\begin{gathered}
F_{V}=d_{V}\left(c_{0}^{V} s_{0}^{V}+c_{1}^{V}\right) \\
F_{V} m_{V}^{2}=d_{V}\left(\frac{\left(s_{0}^{V}\right)^{2} c_{0}^{V}}{2}-c_{2}^{V}\right) \\
F_{V} m_{V}^{4}=d_{V}\left(\frac{\left(s_{0}^{V}\right)^{3}}{3} c_{0}^{V}+c_{3}^{V}\right)
\end{gathered}
$$

Using equations (37) and (38), we determine the values of $F_{V}$ and $s_{0}^{V}$ by assuming the values of $c_{0}^{V}$, with $Q^{2}=s_{0}\left(\alpha_{s}\left(Q^{2} \simeq 1 \mathrm{GeV}^{2}\right)=0.5\right)$ and $c_{1}^{V}$ as calculated in the chiral $\mathrm{SU}(3)$ model. These values are assumed in equation (39) to find the vacuum value of the 4-quark condensate, $c_{3}^{V}$ and hence the value of $\kappa_{i}$.

At finite densities, there is contribution to the spectral function for the vector mesons, due to scattering from the baryons and the equation (27) is modified to

$$
\int_{0}^{\infty} d s e^{-s / M^{2}} R_{p h e n}^{V}(s)+12 \pi^{2} \Pi^{V}(0)=d_{V}\left[c_{0} M^{2}+c_{1}+\frac{c_{2}}{M^{2}}+\frac{c_{3}}{2 M^{4}}\right]
$$


where, in the nuclear medium, $\Pi^{V}(0)=\frac{\rho_{B}}{4 M_{N}}$ for $\mathrm{V}=\omega, \rho$. and vanishes for $\phi$ meson [3, 5, 28, 29]. However, in the presence of hyperons in the hadronic medium, the contribution due to the scattering of the $\omega$ and $\rho$ vector mesons from the baryons is modified to

$$
\Pi^{V}(0)=\frac{1}{4} \sum_{i}\left(\frac{g_{V i}}{g_{V N}}\right)^{2} \frac{\rho_{i}}{M_{i}}
$$

where, $g_{V i}$ is the coupling strength of the vector meson, $\mathrm{V}$ with the $i$-th baryon $(i=$ $\left.N, \Lambda, \Sigma^{ \pm, 0}, \Xi^{-, 0}\right), \rho_{i}$ and $M_{i}$ are the number density and mass of the $i$-th baryon. For the $\omega$ meson, $\frac{g_{\omega i}}{g_{\omega N}}=\left(1, \frac{2}{3}, \frac{2}{3}, \frac{1}{3}\right)$ for $i=N, \Lambda, \Sigma^{ \pm, 0}, \Xi^{-, 0}$ respectively. For the $\rho$ meson, the ratio $\frac{g_{\rho i}}{g_{\rho N}}=(1,0,2,1)$ for $i=\left(N, \Lambda, \Sigma^{ \pm, 0}, \Xi^{-, 0}\right)$. In the nuclear medium, the contribution for the $\phi$ meson due to scattering from nucleons vanishes, since the $\phi$ meson-nucleon coupling strength is zero. In the strange hadronic matter, the contribution is, however, nonzero due to the presence of the hyperons in the medium. For the $\phi$ meson, $\frac{g_{\phi i}}{g_{\phi \Lambda}}=(1,1,2)$ for $i=\left(\Lambda, \Sigma^{ \pm, 0}, \Xi^{-, 0}\right)$.

At finite densities, the finite energy sum rules (FESR) for vacuum given by equations (37) to (39) are modified to

$$
\begin{gathered}
F_{V}^{*}=d_{V}\left(c_{0}^{V} s_{0}^{* V}+c_{1}^{V}\right)-12 \pi^{2} \Pi^{V}(0) \\
F_{V}^{*} m_{V}^{* 2}=d_{V}\left(\frac{\left(s_{0}^{* V}\right)^{2} c_{0}^{V}}{2}-c_{2}^{* V}\right) \\
F_{V}^{*} m_{V}^{* 4}=d_{V}\left(\frac{\left(s_{0}^{* V}\right)^{3}}{3} c_{0}^{V}+c^{* V}{ }_{3}\right)
\end{gathered}
$$

These equations are solved to obtain the medium dependent mass, $m_{V}^{*}$, the scale $s_{0}^{* V}$ and $F_{V}^{*}$, by using the coefficient $k$ of the 4 -quark condensate for the vector mesons, as determined from the FESRs in vacuum.

\section{RESULTS AND DISCUSSIONS}

In this section, we first investigate the effects of density on the scalar gluon condensate and the light quark condensates arising due to the modifications of the dilaton field, $\chi$ and the scalar isoscalar fields $\sigma$ and $\zeta$ calculated in the chiral $\mathrm{SU}(3)$ model. The values of these fields in the isospin asymmetric strange hadronic matter are obtained by solving the coupled equations of these fields in the mean field approximation. The nonstrange and strange quark 

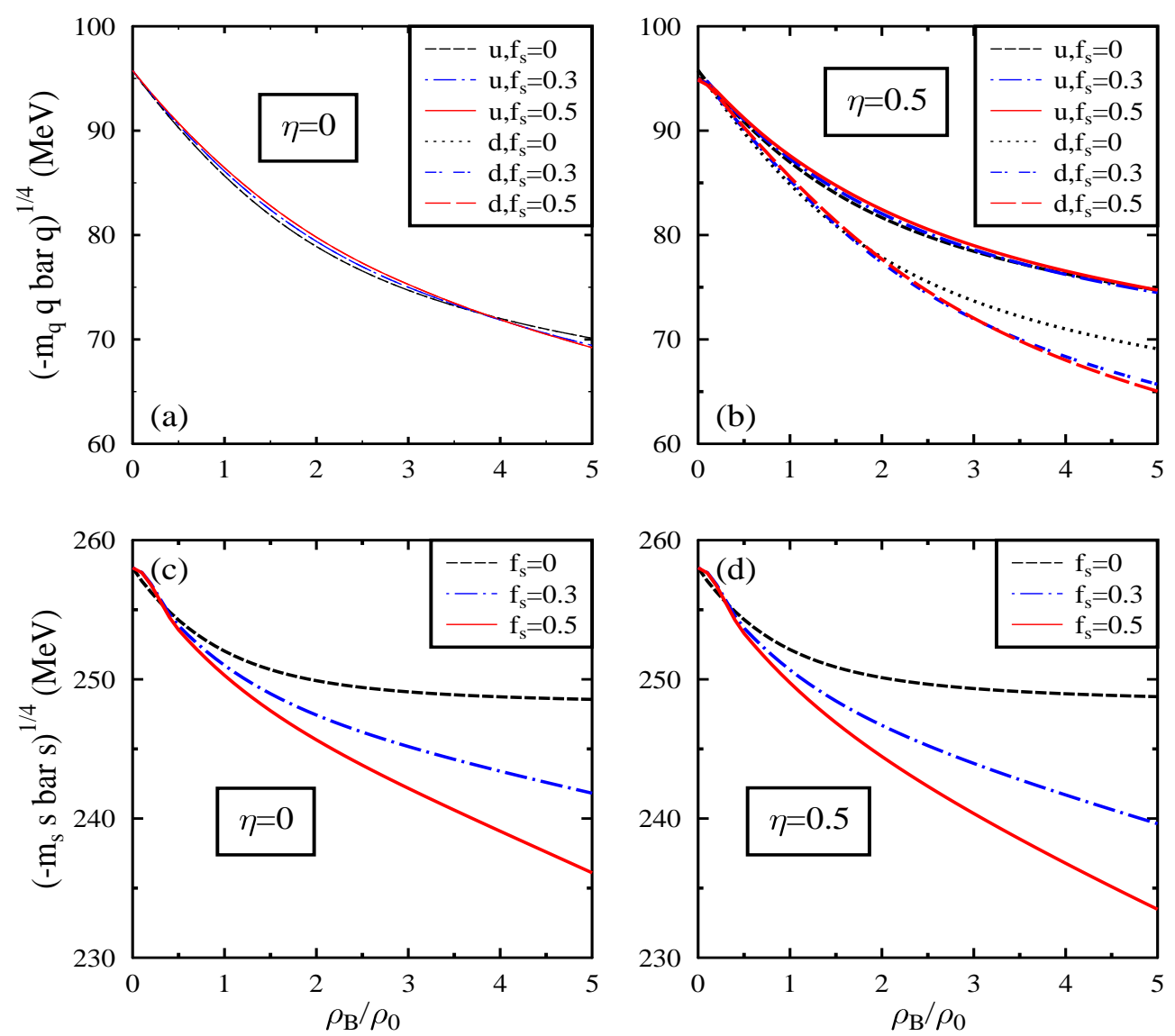

FIG. 1: (Color online) The quark condensates $\left(-m_{q}\langle\bar{q} q\rangle\right)^{1 / 4}(q=u, d)$ and $\left(-m_{s}\langle\bar{s} s\rangle\right)^{1 / 4}$, in units of $\mathrm{MeV}$, are plotted as functions of density for isospin asymmetric hadronic matter (for $f_{s}=0,0.3$ and 0.5 ) in figures (b) and (d), and compared with the isospin symmetric case, shown in subplots (a) and (c). 

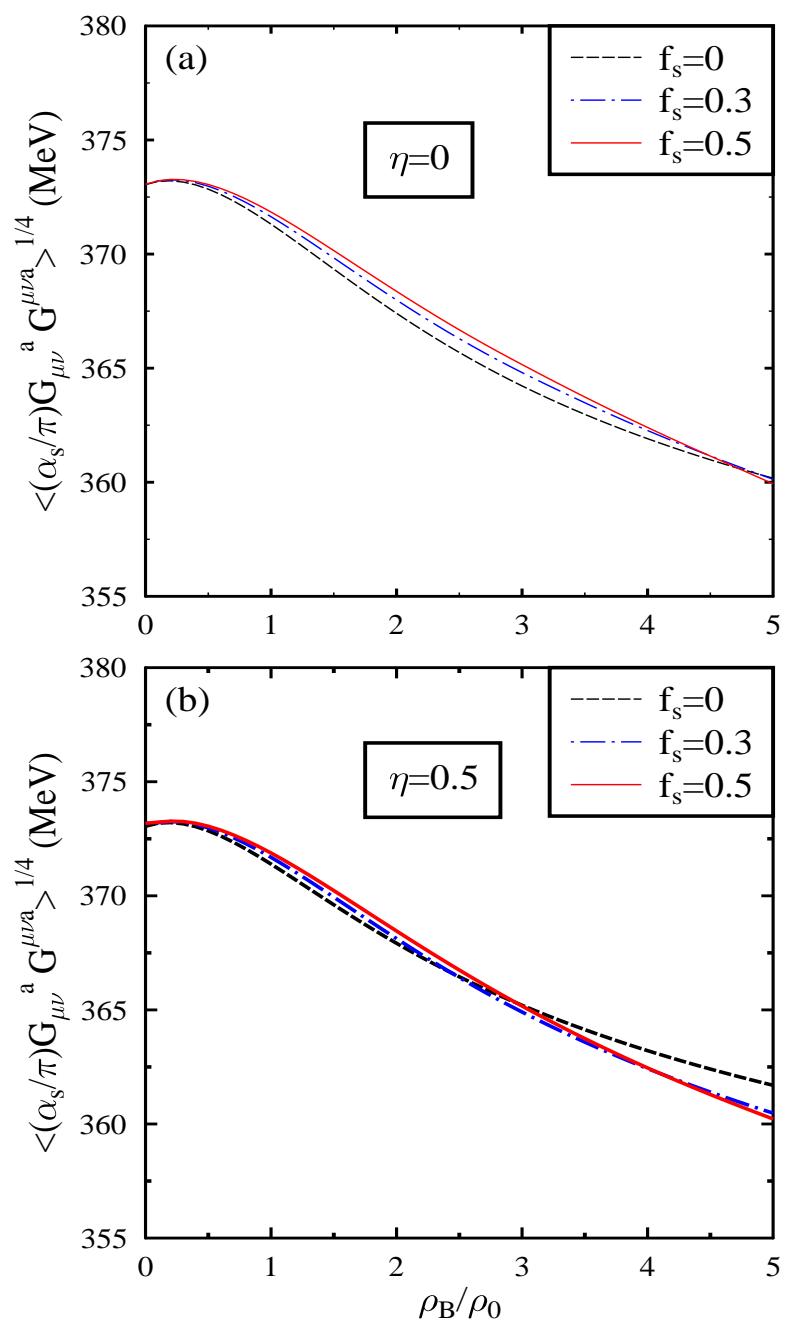

FIG. 2: (Color online) The quantity $\left\langle\frac{\alpha_{s}}{\pi} G^{a}{ }_{\mu \nu} G^{a \mu \nu}\right\rangle^{1 / 4}$ in $\mathrm{MeV}$ plotted as a function of the baryon density in units of the nuclear matter saturation density. This is plotted for isospin asymmetric hadronic matter (for strangeness fraction, $f_{s}=0,0.3,0.5$ and isospin asymmetric parameter, $\eta=0.5$ ) in subplot (b) and compared with the symmetric matter $(\eta=0)$ in subplot (a). 

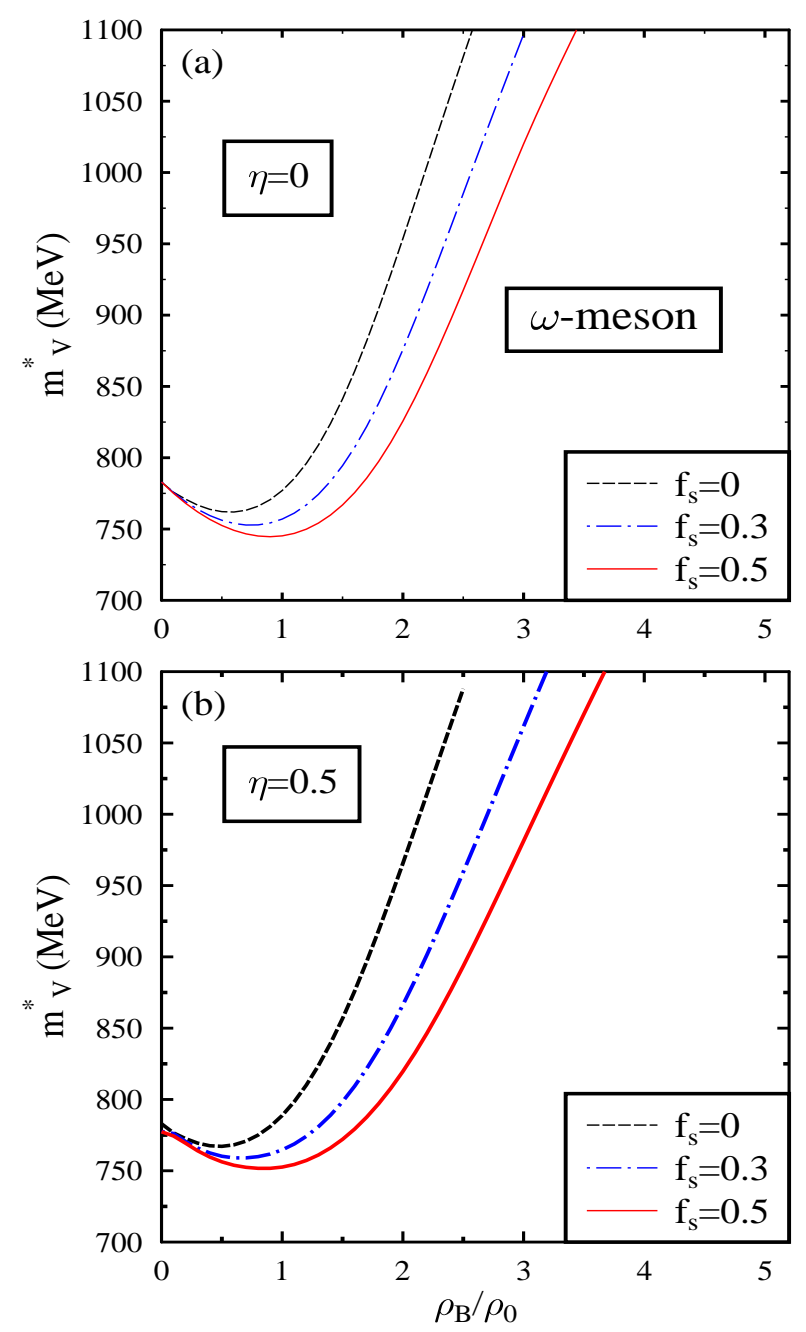

FIG. 3: (Color online) The mass of $\omega$ meson plotted as a function of the baryon density in units of nuclear saturation density, for the isospin asymmetric strange hadronic matter (for strangeness fraction, $f_{s}=0,0.3,0.5$ and isospin asymmetric parameter, $\eta=0.5$ ) in subplot (b) and compared with the symmetric matter $(\eta=0)$ shown in subplot (a). 

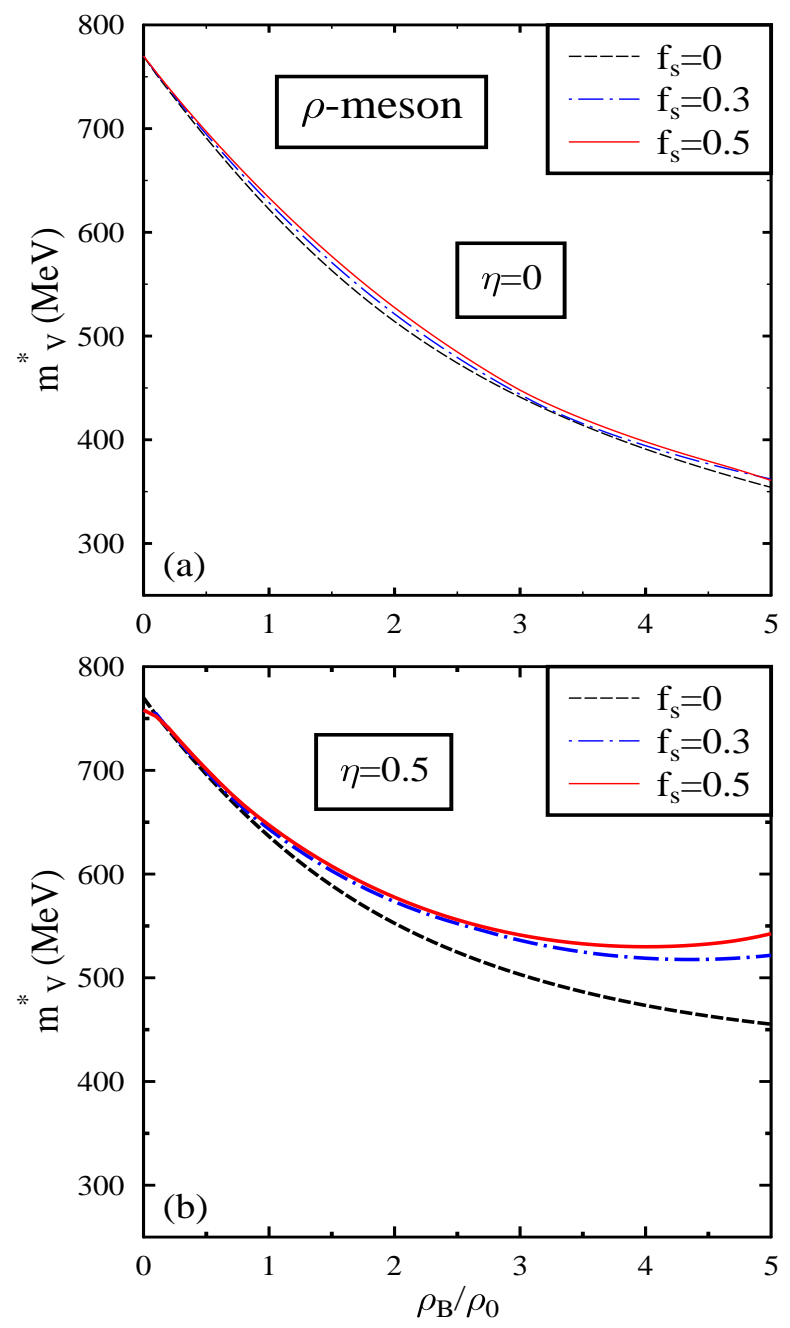

FIG. 4: (Color online) The mass of $\rho$ meson plotted as a function of the baryon density in units of nuclear saturation density, for the isospin asymmetric strange hadronic matter (for strangeness fraction, $f_{s}=0,0.3,0.5$ and isospin asymmetric parameter, $\eta=0.5$ ) in subplot (b) and compared with the symmetric matter $(\eta=0)$ shown in subplot (a). 

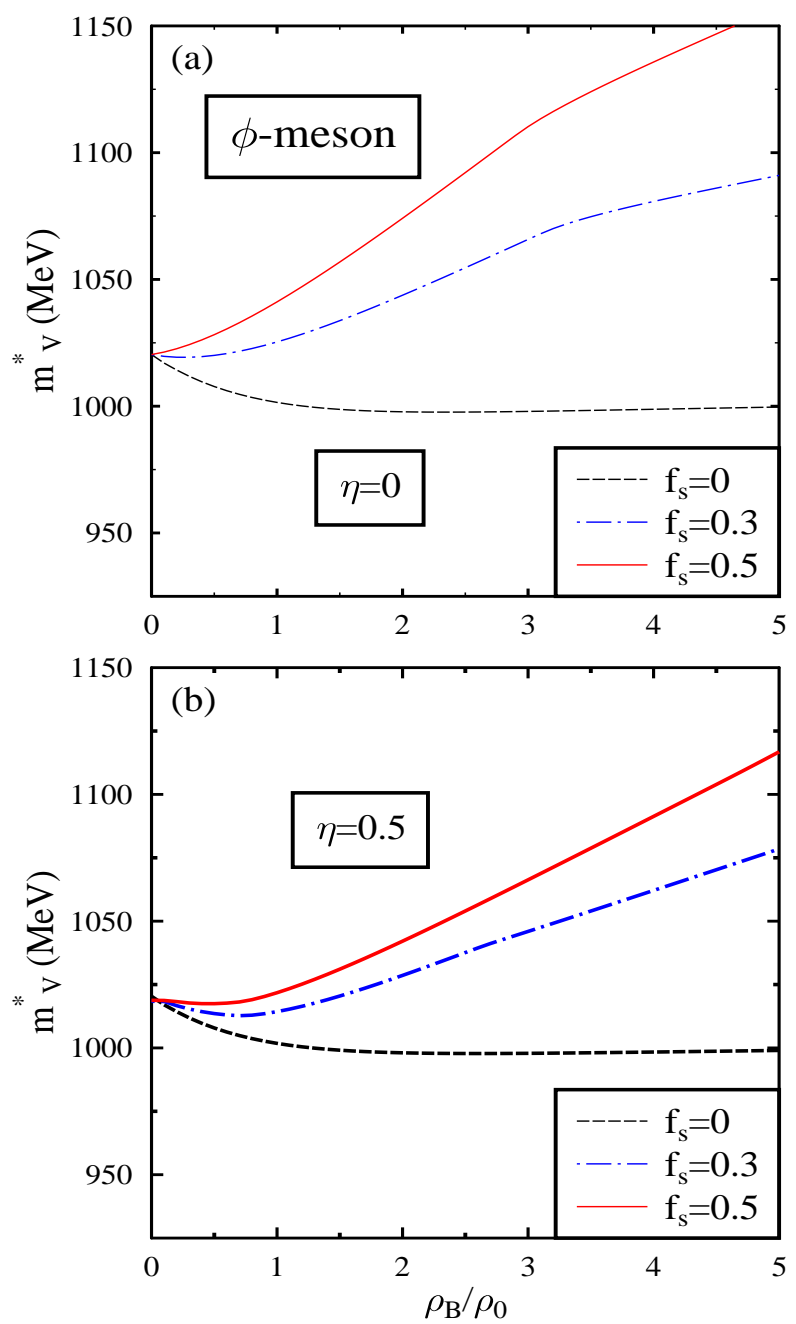

FIG. 5: (Color online) The mass of $\phi$ meson plotted as a function of the baryon density in units of nuclear saturation density, for the isospin asymmetric strange hadronic matter (for strangeness fraction, $f_{s}=0,0.3,0.5$ and isospin asymmetric parameter, $\eta=0.5$ ) in subplot (b) and compared with the symmetric matter $(\eta=0)$ shown in subplot (a). 

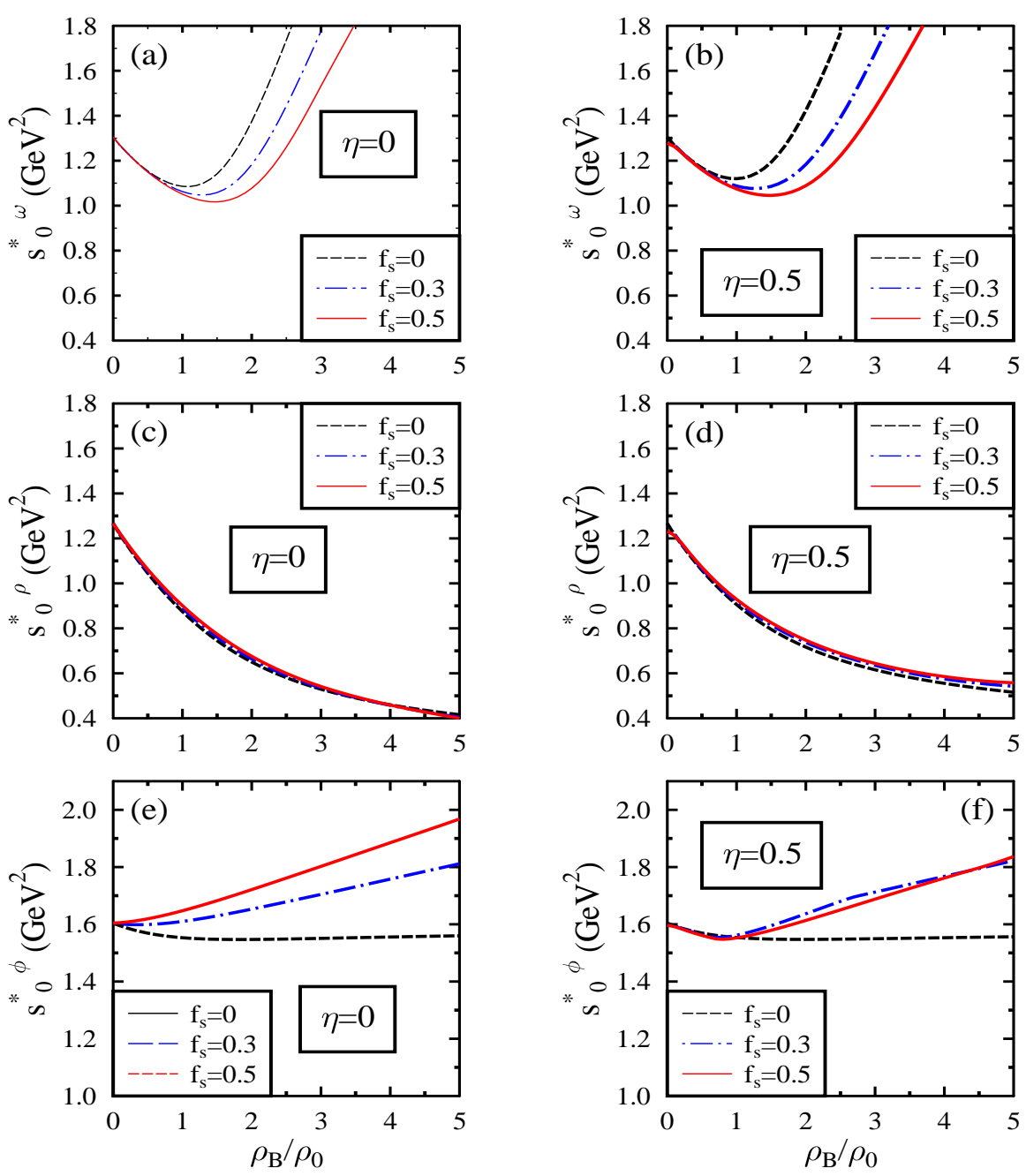

FIG. 6: (Color online) The density dependence of $s_{0}^{* V}$ for the vector mesons $(\omega, \rho$ and $\phi)$ in the strange hadronic matter is shown for the isospin symmetric $(\eta=0)$ and isospin asymmetric (with $\eta=0.5)$ cases for values of $f_{s}=0,0.3$ and 0.5 .

condensates, $\langle\bar{q} q\rangle(q=u, d)$ and $\langle\bar{s} s\rangle$, as well as, the scalar gluon condensate, $\left\langle\frac{\alpha_{s}}{\pi} G^{a}{ }_{\mu \nu} G^{a \mu \nu}\right\rangle$, are calculated from the in-medium values of the fields $\sigma, \zeta$ and $\chi$, by using the equations (7), (8)), (91) and (12), respectively. The values of the current quark masses are taken as $m_{u}=4 \mathrm{MeV}, m_{d}=7 \mathrm{MeV}$ and $m_{s}=150 \mathrm{MeV}$ in the present investigation. In figure 1, we show 

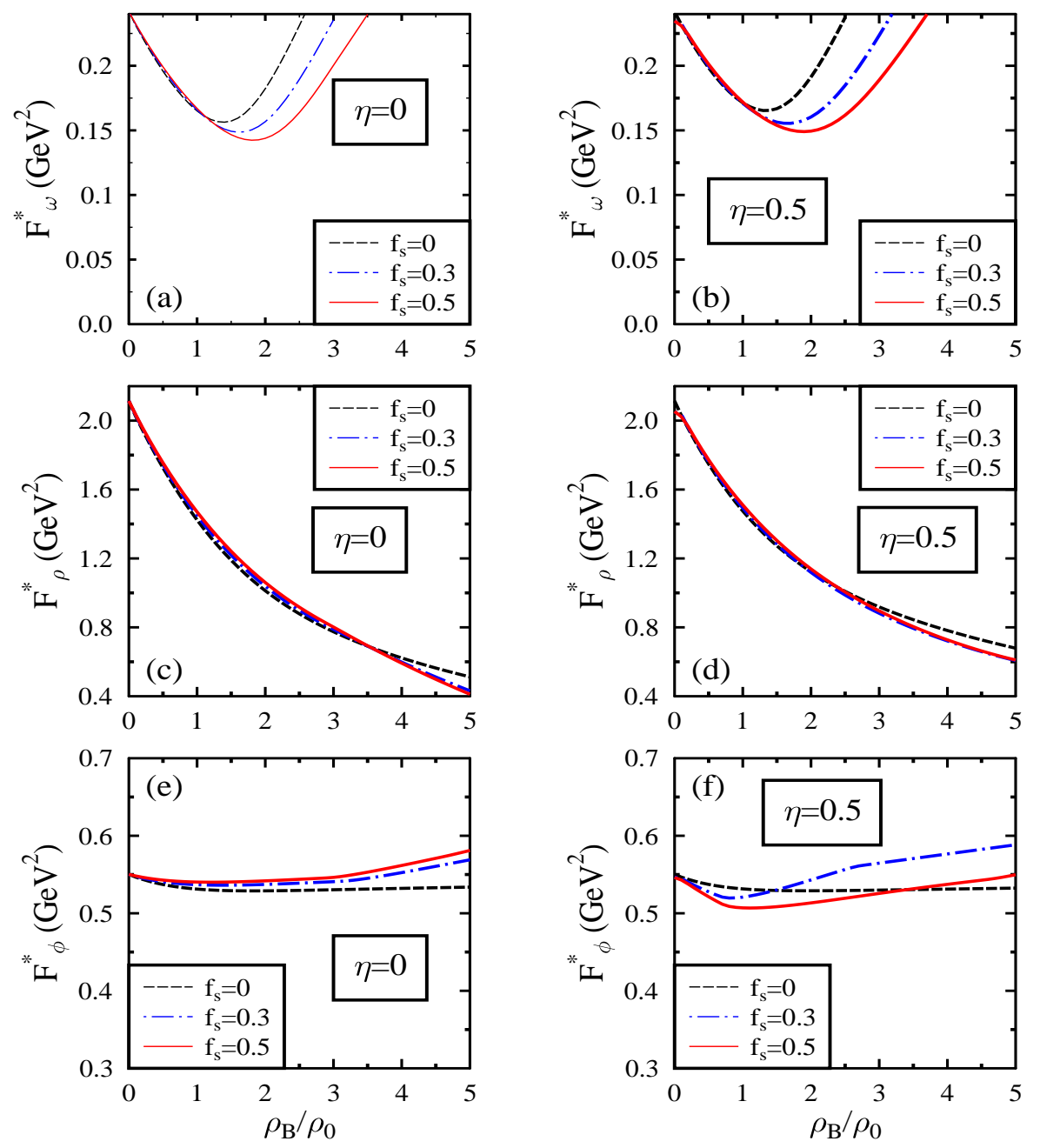

FIG. 7: (Color online) The density dependence of $F_{V}^{*}$ for the vector mesons $(\omega, \rho$ and $\phi)$ in the strange hadronic matter is shown for the isospin symmetric $(\eta=0)$ and isospin asymmetric (with $\eta=0.5)$ cases for values of $f_{s}=0,0.3$ and 0.5 .

the density dependence of the quantities $\left(-m_{q}\langle\bar{q} q\rangle\right)^{1 / 4}(q=u, d),\left(-m_{s}\langle\bar{s} s\rangle\right)^{1 / 4}$ for given isospin asymmetry and strangeness of the hadronic medium. For the isospin symmetric situation $(\eta=0)$, the quantity $\left(-m_{q}\langle\bar{q} q\rangle\right)^{1 / 4}$ is identical for $u$ and $d$ quarks, for a given value of $f_{s}$. It is also seen that the effect from the strangeness fraction is very small. For the 

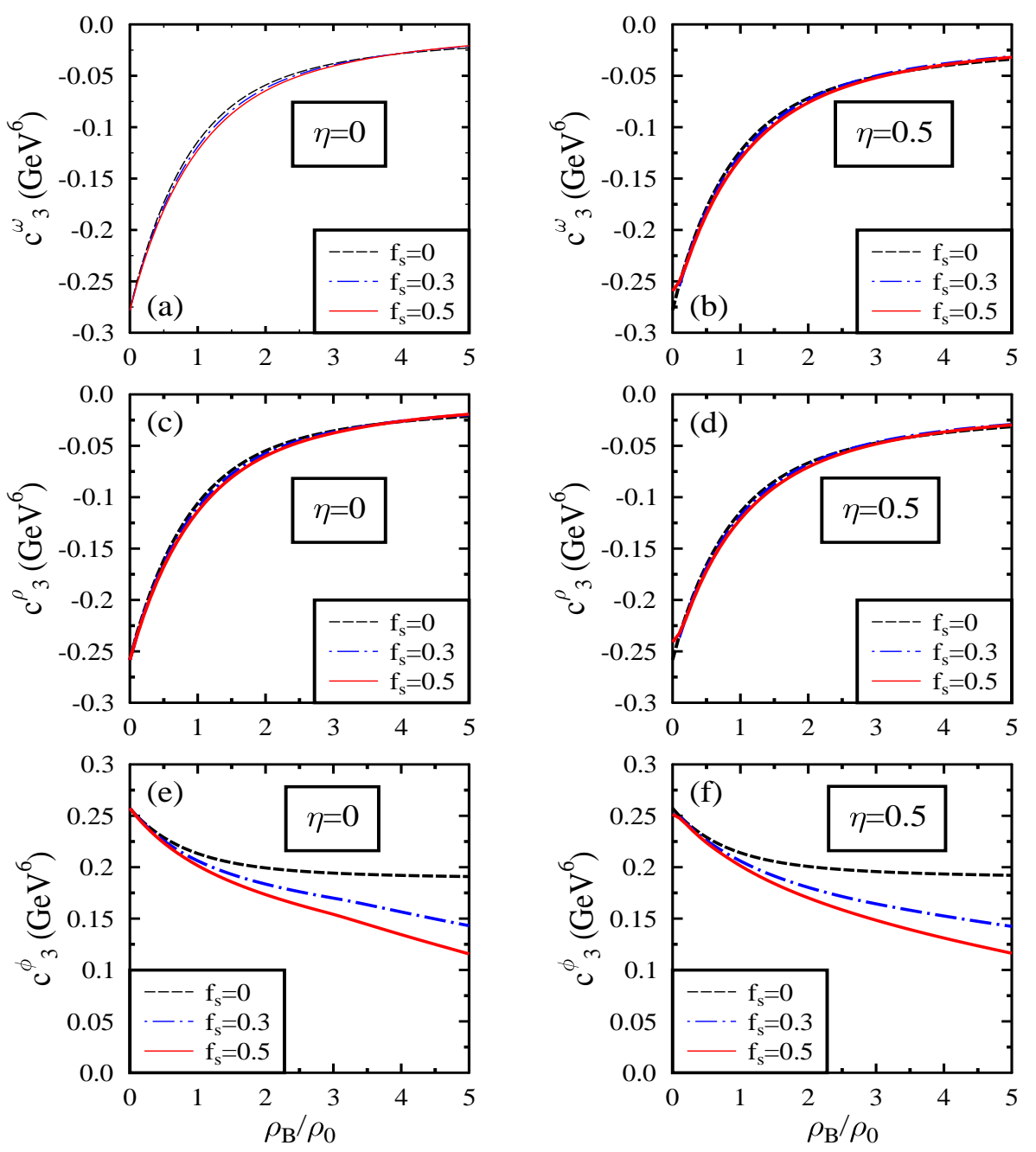

FIG. 8: (Color online) The density dependence of the 4-quark condensate for the cases of the $\omega$, $\rho$ and $\phi$ mesons is shown for the isospin symmetric $(\eta=0)$ and asymmetric (with $\eta=0.5$ ) hadronic matter for the values of the strangeness fraction, $f_{s}=0,0.3$ and 0.5 .

isospin asymmetric situation, the quantities $\left(-m_{u}\langle\bar{u} u\rangle\right)^{1 / 4}$ and $\left(-m_{d}\langle\bar{d} d\rangle\right)^{1 / 4}$ are no longer identical, and their difference is due to the nonzero value of the isoscalar scalar field $\delta$, as can be seen from equations (7) and (8) . For the $u$ quark, there is seen to be smaller drop with density as compared to the $d$ quark due to the negative value of the isoscalar scalar 
field $\delta$ in the medium. For the isospin symmetric nuclear matter, the value of the quantity $\left(-m_{q}\langle\bar{q} q\rangle\right)^{1 / 4}$ for $q=u, d$, changes from the vacuum value of $95.8 \mathrm{MeV}$ to $85.7 \mathrm{MeV}$ at the nuclear matter saturation density. This corresponds to a drop of the quantity, $\left(-m_{q}\langle\bar{q} q\rangle\right)$ for $q=u, d$, by about $36 \%$ at the nuclear matter saturation density from its vacuum value. At a density of $4 \rho_{0}$, this quantity is modified to $(72 \mathrm{MeV})^{4}$, which corresponds to a drop of about $69 \%$ from its vacuum value. The drop of the non-strange condensate in the medium is the dominant contribution to the modification of the $\omega$ and $\rho$ mesons in the medium. The quantity $\left(-m_{s}\langle\bar{s} s\rangle\right)^{1 / 4}$ for given isospin symmetric $(\eta=0)$ and isospin asymmetric (with $\eta=0.5)$ situations are shown in subplots (c) and (d) respectively. The vacuum value of $\left(-m_{s}\langle\bar{s} s\rangle\right)^{1 / 4}$ is about $258 \mathrm{MeV}$, which may be compared with the value of $210 \mathrm{MeV}$ in Ref. [2]. For the symmetric nuclear matter, the quantity $\left(-m_{s}\langle\bar{s} s\rangle\right)^{1 / 4}$ changes from the vacuum value of $258 \mathrm{MeV}$ to $252 \mathrm{MeV}$ and $248.7 \mathrm{MeV}$ at densities of $\rho_{0}$ and $4 \rho_{0}$ respectively, which correspond to about $9 \%$ and $13.7 \%$ drop in the quantity, $8 \pi^{2}\left\langle m_{s} \bar{s} s\right\rangle$ occurring in $c_{2}^{\phi}$ in the finite energy sum rule for the $\phi$ meson given by equation (21). Figure2 shows the quartic root of the scalar gluon condensate, $\left\langle\frac{\alpha_{s}}{\pi} G^{a}{ }_{\mu \nu} G^{a \mu \nu}\right\rangle^{1 / 4}$ as a function of the baryon density in units of the nuclear matter saturation density, for isospin symmetric $(\eta=0)$ as well as asymmetric hadronic medium (with $\eta=0.5$ ) for typical values of the strangeness fraction. The value of the scalar gluon condensate $\left\langle\frac{\alpha_{s}}{\pi} G^{a}{ }_{\mu \nu} G^{a \mu \nu}\right\rangle$ for isospin symmetric nuclear matter is observed to be modified from the vacuum value of $(373 \mathrm{MeV})^{4}$ to $(371.3 \mathrm{MeV})^{4}$ and $(361.9 \mathrm{MeV})^{4}$ at densities of $\rho_{0}$ and $4 \rho_{0}$ respectively, which correspond to about $1.8 \%$ and $11.4 \%$ drop in the medium from its vacuum value. It is thus observed that the light nonstrange quark condensate has a larger drop in the medium as compared to the strange quark condensate as well as the scalar gluon condensate in the medium. This is observed as a much smaller drop of the $\phi$ meson in the medium as compared to the $\omega$ and $\rho$ mesons due to the quark and gluon condensates. The in-medium quark and gluon condensates are used as inputs for the calculations of the vector meson masses in the hadronic medium. The effects of isospin asymmetry as well as strangeness of the medium on the masses of the vector mesons are investigated in the present work. As has already been mentioned, using the vacuum values of the vector meson mass and the quark and gluon condensates, the finite energy sum rules (FESR) for the vector mesons in vacuum given by equations (37), (38) and (39) are solved 
to obtain the values for $s_{0}^{V}, F_{V}$ and the coefficient of the 4-quark condensate, $\kappa_{q(s)}$. The vacuum value of the scale, $s_{0}^{V}$, which separates the resonance part from the continuum part is obtained as $1.3,1.27$ and $1.6 \mathrm{GeV}^{2}$ and the value of $F_{V}$ is obtained as $0.242,0.258$ and $0.55 \mathrm{GeV}^{2}$ for the for $\omega, \rho$ and $\phi$ mesons respectively. The value of the coefficient of the 4-quark condensate is obtained as $7.788,7.236$ and -1.21 for the $\omega, \rho$ and $\phi$ mesons, which are then used to obtain the medium dependent mass, $m_{V}^{*}$, the scale $s_{0}^{* V}$ and $F_{V}^{*}$ for the vector mesons, by solving the FESRs in the medium given by equations (42), (43) and (44).

In figure 3 , the density dependence of the mass of the $\omega$-meson is shown for the cases of isospin symmetric $(\eta=0)$ as well as the asymmetric matter for given values of the strangeness fraction, $f_{s}$. There is seen to be initially a drop in the $\omega$-meson mass with increase in density. However, as the density is further increased, the mass of the $\omega$-meson is observed to increase with density. This behavior can be understood from the equations (42) and (43), which yield the expression for the mass squared of the vector meson as

$$
m_{V}^{* 2}=\frac{\left(\frac{\left(s_{0}^{* V}\right)^{2} c_{0}^{V}}{2}-c_{2}^{* V}\right)}{\left(c_{0}^{V} s_{0}^{* V}+c_{1}^{V}\right)-12 \pi^{2}\left(\Pi^{V}(0) / d_{V}\right)}
$$

The contribution of $c_{1}^{V}$ is negligible for the $\rho$ and $\omega$ mesons, due to the small values of the masses of the $u$ and $d$ quarks. At low densities, the contribution from the scattering of the vector mesons from baryons, given by the last term in the denominator of (45) is negligible and the mass drop of the $\omega$ meson mainly arises due to the drop of the light quark condensates in the medium, given by the second term, $c_{2}^{* V}$ in the numerator which comes with a negative sign. As seen in figure 2, the modification of the scalar gluon condensate of the term $c_{2}^{* V}$ is much smaller than that of the light quark condensate. However, at higher baryon densities, the last term in the denominator, the so-called Landau scattering term, becomes important for the $\omega$ meson. This leads to an increase in the mass of the $\omega$ vector meson with density, as can be observed in figure 3. The denominator becomes negative above a certain value of density, when there does not exist any solution for the mass of the $\omega$ meson, since $m_{V}^{* 2}$ becomes negative. For the case of nuclear matter, the mass of the $\omega$ meson remains very similar in the isospin symmetric as well as isospin asymmetric cases. This is because the modification of the $\omega$ meson at low densities is mainly due to the quark condensates in the combination $\left(m_{u} \bar{u} u+m_{d} \bar{d} d\right)$, which depends only on the value of $\sigma$ (as seen from equations (7) and (8) ), and, $\sigma$ is marginally different for the symmetric and 
asymmetric cases. At higher densities, the effect of the Landau scattering term becomes important. However, there is still observed to be very small difference between the $\eta=0$ and $\eta=0.5$ cases of nuclear matter, since the dependence of this term on the proton and neutron densities is in the form $\left(\rho_{p}+\rho_{n}\right)$, which is same for the two cases at a given density. With the inclusion of hyperons in the medium, the contribution of the scattering term in the denominator of equation (45), becomes smaller in magnitude due to the smaller values of the baryon- $\omega$ meson coupling strengths for the hyperons as compared to the nucleons. However, the trend of the initial mass drop followed by an increase at higher densities is still seen to be the case for the mass of the $\omega$-meson. However, the density above which the $\omega$-mass is observed to increase with density, is seen to be higher for finite strangeness fraction in the hadronic medium, since the contribution from the Landau scattering term is smaller for the case of hyperonic matter as compared to nuclear matter. For the $\rho$ meson, the contribution from the Landau scattering term remains small as compared to the contribution from the light quark condensate in the medium, due to the factor $\left(1 / d_{V}\right)$ in this term, which makes the contribution of the Landau scattering term to be 9 times smaller than that of the $\omega$ meson, as $\left(1 / d_{\rho}\right) /\left(1 / d_{\omega}\right)=9$. This is observed as a monotonic decrease of the mass of the $\rho$-meson with density, in figure 4. The effects of the strangeness fraction as well as isospin asymmetry of the medium are seen to be small on the $\rho$ meson mass. In figure 5. the mass of $\phi$ meson is plotted as a function of the baryon density in units of nuclear matter saturation density, for isospin symmetric and asymmetric cases for typical values of the strangeness fraction. Due to the larger value of the strange quark mass as compared to the $u(d)$ quark masses, the contribution from $c_{1}^{\phi}$ is no longer negligible as was the case for $\omega(\rho)$ meson. The dominant contribution to the mass modification of the $\phi$ meson is from the in-medium modification of the strange quark condensate of the Wilson coefficient $c_{2}^{\phi}$ in the nuclear medium. This is because the $\phi$-meson has no contribution from the scattering term in nuclear matter, since the nucleon- $\phi$ meson coupling is zero. The strange quark condensate as well as the scalar gluon condensate have very small effects from isospin asymmetry, leading to the modifications of the $\phi$ meson mass to be very similar in the isospin symmetric and asymmetric nuclear matter. There are, however, contributions from the Landau scattering term due to the hyperons in the medium for nonzero $f_{s}$, which leads to an increase in the 
mass of the $\phi$ meson at higher values of the densities. For nuclear matter, the mass of the $\phi$ meson does not have contribution from the scattering term and since the in-medium modifications of both the strange quark condensate as well as the scalar gluon condensate are small and occur with opposite signs in the coefficient $c_{2}^{* \phi}$, the mass of $\phi$ meson is observed to have negligible change with density, the value being modified from the vacuum value of 1020 $\mathrm{MeV}$ to about $1000 \mathrm{MeV}$ at a density of $5 \rho_{0}$. For the case of isospin symmetric hyperonic matter, there is seen to be an increase in the mass of the $\phi$ meson at low densities, due to scattering from the $\Xi^{-}$and $\Xi^{0}$, whose number densities are equal for this $\eta=0$ case. The $\Sigma^{+}, \Sigma^{-}$and $\Sigma^{0}$ (with equal number densities) start appearing at around $3 \rho_{0}$, when the number densities of the $\Xi^{-}$and $\Xi^{0}$ show a downward trend with density. It is the overall contributions from the hyperons to the scattering term which leads to the observed increase in the mass of the $\phi$ meson in the strange hadronic medium with $f_{s}=0.3$ and 0.5 , shown in figure 5. For the isospin asymmetric hyperonic matter, there is contribution from the $\Sigma^{+}$ and $\Xi^{0}$ for $\eta=0.5$ situation (but not from $\Sigma^{0,-}$ and $\Xi^{-}$), which is seen as a smaller increase of the $\phi$ mass at high densities as compared to the isospin symmetric hyperonic matter.

The density dependence of the scale $s_{0}^{* V}$, which separates the resonance part from the perturbative continuum, is shown in figure 6 for the $\omega, \rho$ and $\phi$ vector mesons. For isospin symmetric nuclear matter, for the $\omega$ meson, the vacuum value of $1.3 \mathrm{GeV}^{2}$ is modified to about 1.086 and $1.375 \mathrm{GeV}^{2}$ at densities of $\rho_{0}$ and $2 \rho_{0}$ respectively. The dependence of $s_{0}^{* V}$ on density as an initial drop followed by an increase is similar to that of the density dependence of the mass of the $\omega$ meson. This can be understood in the following way. From the medium dependent FESRs, we obtain the expression for the scale $s_{0}^{* V}$ as

$$
s_{0}^{* V}=m_{V}^{* 2}\left(1+\frac{2}{m_{V}^{*} c_{0}}\left(c_{1}^{V} m_{V}^{*}{ }^{2}+c_{2}^{* V}-\left(12 \pi^{2} \Pi(0) / d_{V}\right)\right)\right)^{1 / 2} .
$$

The value of the second term in the bracket, within the square root, is found to be small as compared to 1. At higher densities, the second term still remains small as compared to 1, due to the cancelling effect of the contributions from the quark condensate and the Landau scattering term. This is seen as the density dependence of $s_{0}^{* \omega}$ to have first a drop and then an increase with density as found for the mass of the $\omega$ meson. The dependence of the scale $s_{0}^{* V}$ for the $\rho$ meson is observed to be a monotonic drop with increase in density, due to the negligible contribution from the Landau damping term as compared to the contribution 
from the light quark condensate. In the case of $\phi$ meson, the effect of the scattering term is zero for the nuclear matter case, when $s_{0}^{* \phi}$ is observed to have a small drop due to the marginal drop of the strange condensate and the gluon condensate in the medium. For the hyperonic matter, there is observed to be an increase in $s_{0}^{* \phi}$ due to the scattering from the hyperons, which is observed to be larger for the isospin symmetric case as compared to the isospin asymmetric situation. In figure 7 , the value of $F_{V}^{*}$ is plotted as a function of density. From the first finite energy sum rule given by equation (42), due to the small masses of the $u$ and $d$ quarks, the term $c_{1}^{V}$ is negligible for the $\omega$ and $\rho$ mesons. At low densities, the value of $F_{V}^{*}$ turns out to be proportional to $s_{0}^{* V}$, since the contribution from the Landau scattering term is small. At higher densities, there is contribution from the Landau scattering term, which modifies the behavior of $F_{V}^{*}$ to a slower change with density for the $\omega$ meson. For the $\rho$ meson, this is approximately proportional to $s_{0}^{* \rho}$ as the Landau term has negligible contribution. For the $\phi$ meson, the scattering from the hyperons leads to an increase of $F_{\phi}^{*}$ at higher densities. In figure 8, we have plotted the quartic quark condensate, $c_{3}^{V}$ for the $\omega, \rho$ and $\phi$ mesons, given by equations (34) and (35) as functions of density, for the isospin symmetric and asymmetric nuclear (hyperonic) matter. For the $\rho$ and $\omega$ mesons, the values of $\kappa$ calculated from the vacuum FESRs are found to be 7.236 and 7.788 , which yield very similar values for the 4-quark condensate for the $\omega$ and $\rho$ mesons, shown in figure 8, The vacuum FESRs for the $\phi$ meson yield the 4-quark condensate to be negative, with the value of $\kappa$ as -1.21 . There is seen to be a large effect from the strangeness fraction of the medium on $c_{3}^{\phi}$, since the strange condensate has appreciable effect from $f_{s}$, as can be seen from figure 1 .

\section{SUMMARY}

In the present investigation, we have calculated the effect of density on the masses of the light vector mesons $(\omega, \rho$ and $\phi)$ using the QCD sum rules, from the light quark condensates and gluon condensates in the medium calculated within a chiral SU(3) model. The effects of the isospin asymmetry as well as the strangeness of the medium on the modifications of these masses have also been investigated. The light quark condensates $(\langle\bar{u} u\rangle,\langle\bar{d} d\rangle,\langle\bar{s} s\rangle)$ in the isospin asymmetric strange hadronic medium are calculated from the values of the 
nonstrange and strange scalar mesons, $\sigma$ and $\zeta$, and the isoscalar scalar meson, $\delta$ of the explicit symmetry breaking term of the chiral $\mathrm{SU}(3)$ model. The scalar gluon condensate is calculated from a scalar dilaton field, which is introduced in the chiral SU(3) model to mimic the scale symmetry breaking of QCD. The mass of the $\omega$ meson is observed initially to drop with increase in density in the hadronic matter. This is because the magnitudes of the light nonstrange quark condensates become smaller in the hadronic medium as compared to the values in vacuum. However, as the density is further increased, there is seen to be a rise in its mass, when the effect from the Landau term due to the scattering of the $\omega$ meson from the baryons becomes important. In the presence of hyperons, the increase in the mass of the $\omega$ meson occurs at a higher value of the density as compared to the case of nuclear matter. This is because the contribution from the Landau term becomes less with inclusion of hyperons due to smaller values of the coupling strengths of the $\omega$ meson with hyperons as compared to coupling strengths with the nucleons. The $\rho$ meson mass is observed to drop monotonically with density dominantly from the drop in the light quark condensate in the medium, with negligible contribution from the Landau scattering term. The effect of isospin asymmetry is observed to be small on the masses of the $\omega$ and $\rho$ mesons, as the dependence on the light quark condensates is through the combination $\left(m_{u} \bar{u} u+m_{d} \bar{d} d\right)$, which has marginal effect from the isospin asymmetry. For the $\phi$ meson, there is observed to be a drop in the mass in nuclear matter due to the modification of the strange quark condensate and scalar gluon condensate, because the contribution from the Landau term for the $\phi$ meson vanishes in the nuclear matter. The mass shift of $\phi$ meson in nuclear medium is seen to be small, of the order of $20 \mathrm{MeV}$ at a density of $5 \rho_{0}$. This is because the strange condensate as well as gluon condensate have very small modification in the medium and occur with opposite signs in the coefficient $c_{2}^{* \phi}$. In the presence of hyperons, however, there is seen to be an increase in the mass of the $\phi$ meson with density due to contribution from the Landau term arising from scattering of the $\phi$ meson with the hyperons. The mass of the $\phi$ meson is observed to have larger effect from the Landau scattering term for the isospin symmetric case as compared to the isospin asymmetric hyperonic matter. 


\section{Acknowledgments}

The author acknowledges financial support from Department of Science and Technology, Government of India (project no. SR/S2/HEP-031/2010).

[1] R. Rapp and J. Wambach, Adv. Nucl. Phys. 25, 1 (2000).

[2] T. Hatsuda, S.H. Lee, Phys. Rev. C 46, (1992) R34.

[3] T. Hatsuda, S.H. Lee, H. Shiomi, Phys. Rev. C 52, (1995) 3364.

[4] S. Zschocke, O. P. Pavlenko and B. Kämpfer, Eur. Phys. Jour. A 15, 529 (2002).

[5] F.Klingl, N. Kaiser, W. Weise, Nucl. Phys. A 624,527 (1997).

[6] Y. Kwon, M. Procura and W. Weise, Phys. Rev. C 78, 055203 (2008).

[7] A.K. Dutt-Mazumder, R. Hofmann, M. Pospelov, Phys. Rev. C 63, 015204 (2000).

[8] A. Mishra, K. Balazs, D. Zschiesche, S. Schramm, H. Stöcker and W. Greiner, Phys. Rev. C 69, $024903(2004)$.

[9] P. Papazoglou, D. Zschiesche, S. Schramm, J. Schaffner-Bielich, H. Stöcker, and W. Greiner, Phys. Rev. C 59, 411 (1999).

[10] A. Mishra, A. Kumar, S. Sanyal, V. Dexheimer, S. Schramm, Eur. Phys. Jour. A 45, 169 (2010).

[11] D. Zschiesche, A. Mishra, S. Schramm, H. Stöcker and W. Greiner, Phys. Rev. C 70, 045202 (2004).

[12] A. Mishra, E. L. Bratkovskaya, J. Schaffner-Bielich, S. Schramm and H. Stöcker, Phys. Rev. C 70, 044904 (2004). A. Mishra and S. Schramm, Phys. Rev. C 74, 064904 (2006).

[13] Amruta Mishra, Arvind Kumar, Sambuddha Sanyal, S. Schramm, Eur. Phys, J. A 41, 205 (2009).

[14] Amruta Mishra and Arindam Mazumdar, Phys. Rev. C 79, 024908 (2009).

[15] A. Mishra, E. L. Bratkovskaya, J. Schaffner-Bielich, S.Schramm and H. Stöcker, Phys. Rev. C 69, $015202(2004)$.

[16] Arvind Kumar and Amruta Mishra, Phys. Rev. C 81, 065204 (2010).

[17] Arvind Kumar and Amruta Mishra, Eur. Phys. J. A 47, 164 (2011). 
[18] S.Weinberg, Phys. Rev. 1661568 (1968).

[19] S. Coleman, J. Wess, B. Zumino, Phys. Rev. 1772239 (1969); C.G. Callan, S. Coleman, J. Wess, B. Zumino, Phys. Rev. 1772247 (1969).

[20] W. A. Bardeen and B. W. Lee, Phys. Rev. 1772389 (1969).

[21] J. Schechter, Phys. Rev. D 21, 3393 (1980).

[22] J.Ellis, Nucl. Phys. B 22, 478 (1970); B. A. Campbell, J. Ellis and K. A. Olive, Nucl. Phys. B 345, 57 (1990).

[23] Erik K. Heide, Serge Rudaz and Paul J. Ellis, Nucl. Phys. A 571, (2001) 713.

[24] Arvind Kumar and Amruta Mishra, Phys. Rev. C 82, 045207 (2010).

[25] Thomas D. Cohen, R. J. Furnstahl and David K. Griegel, Phys. Rev. C 45, 1881 (1992).

[26] M. A. Shifman, A. I. Vainshtein and V. I. Zakharov, Nucl. Phys. Bb 147, 385 (1979).

[27] M. A. Shifman, A. I. Vainshtein and V. I. Zakharov, Nucl. Phys. Bb 147, 448 (1979).

[28] A. I. Bochkarev and M. E. Shaposhnikov, Phys. Lett. B 145, 276 (1984); ibid, Nucl. Phys. B 268, 220 (1986).

[29] W. Florkowski, W. Broniowski, Nucl. Phys. A 651, 397 (1999). 